\title{
The visualization of flow paths in experimental studies of clay-rich materials
}

\author{
A. C. Wiseall ${ }^{*}$, R. J. Cuss, C. C. Graham and J. F. Harrington \\ British Geological Survey, Keyworth, Nottingham NG12 5GG, UK
}

[Received 10 October 2014; Accepted 17 May 2015; Associate Editor: Nicholas Bryan]

\section{ABSTRACT}

One of the most challenging aspects of understanding the flow of gas and water during testing in clay-rich low-permeability materials is the difficulty in visualizing localized flow. Whilst understanding has been increased using X-ray Computed-tomography (CT) scanning, synchrotron X-ray imaging and Nuclear Magnetic Resonance (NMR) imaging, real-time testing is problematic under realistic in situ conditions confining pressures, which require steel pressure vessels. These methods tend not to have the nano-metre scale resolution necessary for clay mineral visualization, and are generally not compatible with the long duration necessary to investigate flow in such materials. Therefore other methods are necessary to visualize flow paths during post-mortem analysis of test samples. Several methodologies have been established at the British Geological Survey (BGS), in order to visualize flow paths both directly and indirectly. These include: (1) the injection of fluorescein-stained water or deuterium oxide; (2) the introduction of nanoparticles that are transported by carrier gas; (3) the use of radiologically tagged gas; and (4) the development of apparatus for the direct visualization of clay. These methodologies have greatly increased our understanding of the transport of water and gas through intact and fractured clay-rich materials. The body of evidence for gas transport through the formation of dilatant pathways is now considerable. This study presents observations using a new apparatus to directly visualize the flow of gas in a kaolinite paste. The results presented provide an insight into the flow of gas in clay-rich rocks. The flow of gas through dilatant pathways has been shown in a number of argillaceous materials (Angeli et al., 2009; Autio et al., 2006; Cuss et al., 2014; Harrington et al., 2012). These pathways are pressure induced and an increase in gas pressure leads to the dilation of pathways. Once the gas breakthrough occurs, pressure decreases and pathways begin to close. This new approach is providing a unique insight into the complex processes involved during the onset, development and closure of these dilatant gas pathways.

KEYworDs: gas flow, pathway dilation, kaolinite, pathway visualization, geological disposal.

\section{Introduction}

Sound understanding of the mechanisms involved in advective gas flow through clay-rich materials is essential to the safe disposal of radioactive waste in

* E-mail: andyw@bgs.ac.uk

DOI: 10.1180/minmag.2015.079.06.09 a Geological Disposal Facility (GDF). Disposal concepts may use clay-rich materials as a host rock and/or engineered barriers. Disposal may also occur in salt or crystalline host rocks; however clay-rich materials may still be part of the engineering barrier. Clay-rich materials are an attractive option for use in the disposal concept due to their low permeabilities, ability to adsorb radionuclides and their self-sealing nature. Typical candidate
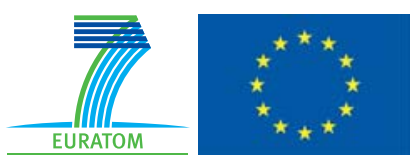

The publication of this research has been funded by the European Union's European Atomic Energy Community's (Euratom) Seventh Framework programme FP7 (2007-2013) under grant agreements $n^{\circ} 249396$, SeclGD, and $n^{\circ} 323260$, SecIGD2. 
materials have very low permeabilities (in the range $10^{-12}-10^{-15} \mathrm{~m} / \mathrm{s}$ ) which are derived from their large clay content and nano-scale pore networks which are often highly discontinuous in nature.

Within a GDF, gas will be generated by anaerobic corrosion of components of the engineered barrier (e.g. iron insert within copper canisters), radiolysis of groundwater and degradation of organic matter which produces hydrogen, methane and carbon dioxide (Ortiz et al., 2002). If the rate at which this gas is produced exceeds the diffusional capability of the clay-rich engineered barrier and/or host rock, a consequent build-up in pressure will occur. This increase in pressure over time may exceed a critical level, at which point gas enters the material, potentially also transporting harmful radionuclides (Weetjens and Sillen, 2006; Ortiz et al., 2002; Wikramaratna et al., 1993). The mechanism allowing gas flow, its potential spatial distribution and temporal evolution, will strongly control the likelihood of radionuclide transport. Understanding these aspects of gas flow in clay-rich materials is therefore crucial for the assessment of repository performance.

To forecast the movement of gas through these materials, it is first vital to define the appropriate model that best fits the underlying physics governing flow. Gas movement through clay-rich materials may occur by four mechanisms (Cuss et al., 2014; Marschall et al., 2005): (1) gas movement by diffusion and/or solution, which is governed by Fick's and Henry's laws respectively; (2) gas migration via visco-capillary flow, governed by Darcy's law; (3) gas flow through localized dilatant pathways; the flow along these pathways is controlled by a combination of gas pressure and the state of effective stress; (4) gas flow along macro fractures, which form in similar ways to the way in which a hydro fracture may form in a hydrocarbon reservoir for reservoir stimulation.

Diffusion (1) will always occur and gas fracture (4) will only occur if gas generation rates are very high. In recent years experimental studies have concentrated on the advective flow of gas and the likelihood of visco-capillary flow (2) or dilatant pathway formation (3) being the primary mechanism for gas movement.

There is a growing body of evidence that in the case of plastic clays the classic concept of twophase flow in porous media cannot always be applied (Horseman et al., 1999). Flow through dilatant pathways has been shown in a number of experiments on argillaceous materials (Angeli et al., 2009; Autio et al., 2006; Cuss et al., 2014; Graham et al., 2012; Harrington et al., 2012).
These pathways are pressure-induced and their propagation is controlled by the internal gas pressure and the structural properties of the clay. The exact form, distribution and underlying physics behind the formation of these pathways are yet to be fully described. This has led to the development of experimental apparatuses and methodologies for the direct characterization of gas pathways, e.g. by injecting nano particles within the gas, combined with scanning electron microscopy to locate the nano particles post-test (Harrington et al., 2012).

In recent years techniques such as X-ray CT scanning, synchrotron X-ray imaging and Nuclear Magnetic Resonance (NMR) imaging have been applied to the studies of gas movement in argillaceous materials (Beseulle et al., 2013; Chen et al., 2014). However, these methods tend not to have the nano-metre scale necessary for clay mineral visualization, and are generally not compatible with the long duration necessary to investigate flow in argillaceous materials. As such, other methods are necessary in order to visualize the development of pathways.

With this in mind a new experimental apparatus has been designed and manufactured by the British Geological Survey (BGS) with the aim of visualizing and recording the development of pathways in clay-rich materials. This paper presents details on the experimental apparatus and methodology, along with qualitative results from preliminary testing and observations on gas flow in clay-rich materials.

\section{Methodology}

The Fracture Visualization Rig (FVR) was designed and manufactured by the Transport Properties Research Laboratory at the BGS. The FVR (Fig. 1 $1 a, b$ ) consists of a $110 \mathrm{~mm}$ diameter, $50 \mathrm{~mm}$ thick fused silica glass window held rigidly in place by a $230 \mathrm{~mm}$ diameter steel collar. Twelve torqued bolts hold the collar to a $250 \mathrm{~mm}$ diameter steel plate and can impose a normal load of up to $3 \mathrm{MPa}$. The bolts are spaced at $30^{\circ}$ intervals and each is tightened to a known torque. A thin clay paste is placed, as evenly as possible, between the lower steel platen and the glass window and is held laterally in place by a porous plastic filter. A paper gasket is placed between the steel collar and the glass window to protect the glass. Gas is injected, at the same constant rate in each test, into the clay paste at the centre of the lower platen; in these experiments helium was used as permeant. The gas was injected and controlled, at a rate of $100 \mathrm{ml} / \mathrm{hr}$, 

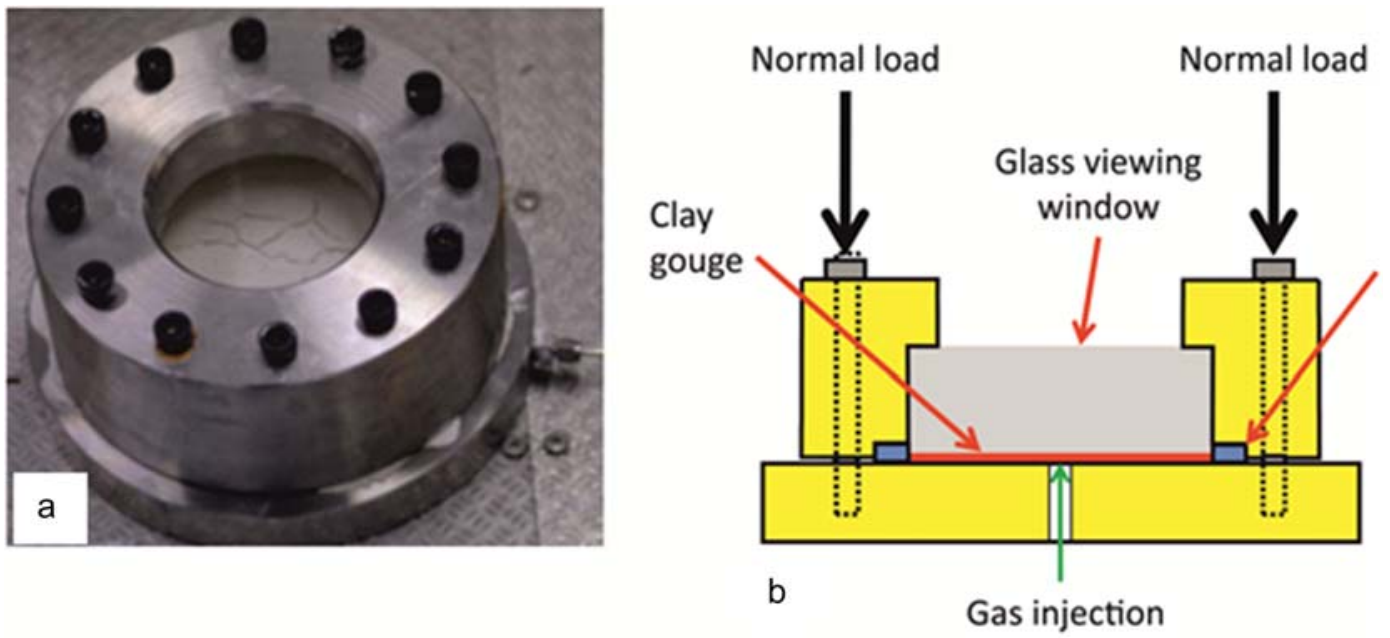

Filter

FIG. 1. Experimental set-up of the FVR. (a) Photo of FVR set-up; (b) Schematic cross-section showing arrangement of apparatus and point of gas injection into the clay paste.

by a high precision Teledyne ISCO-260D syringe pump which was connected to a gas-water interface vessel. This set-up allows a gas injection pressure ranging from 0.5 to $15 \mathrm{MPa}$. A Nikon Digital Single-Lense Reflex camera captured images every three seconds, with images combined to create a time-lapse video of each test. The images are then processed using Adobe Lightroom software, where the images are inverted and then converted to greyscale, this provides more clarity to the images.

A total of six tests have been performed using a pure kaolinite clay powder, mixed with deionized water to give a gravimetric water content of $80 \%$ (following Sathar et al., 2012). For these tests a normal load of $1 \mathrm{MPa}$ was applied to the clay paste. The results of three of the six tests are presented and discussed here, although all six tests showed consistent results.

\section{Results}

Early results from the FVR have shown that it is capable of imaging the development of pathways in clay rich materials, whilst subject to in situ stresses. Findings are presented in terms of images captured during testing, showing key characteristics from each test. Gas is injected through the base of the central plate with images captured throughout the complete test duration. Results from the three tests are shown below; comparisons and similarities between the images are also discussed.

Figure 2 shows the first image following gas entry from each test. All tests were prepared using the same method and also took place at the same pressure conditions. The ring in Fig. $2 a$ shows the position of the gas injection port beneath the clay.

Figure $2 a$ for Test FVR_K01 shows that gas entered the paste in two main areas. The timing on this image shows that this occurs only $15 \mathrm{~s}$ after gas injection began. Gas pressure at the injection port results in the compression of the clay paste (piston effect). This means that gas movement away from the injection point tended to occur along the lower platen and this explains why gas pathways are not observed at the injection point, highlighted in Fig. 2. The distribution of gas pathways throughout the clay paste is not homogeneous and occurs in isolated areas. Figure $2 b$ shows the first image where pathways have developed in Test FVR_K02; this occurs $87 \mathrm{~s}$ after gas injection began. The pathways are all isolated, with no interlinking, as observed in Fig. $2 a$. Figure $2 c$ shows the first pathway development in Test FVR_K03; this occurs after $132 \mathrm{~s}$. These pathways form to the right of the injection port in two areas. The larger more defined area has a very irregular edge and also appears to have begun to develop into a larger pathway. 


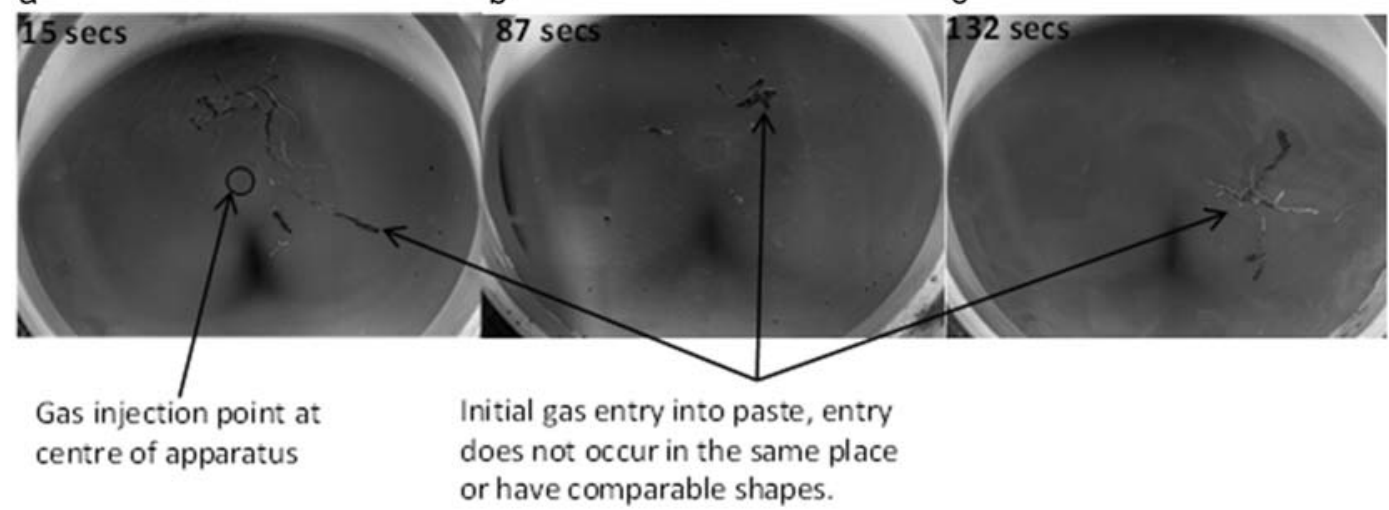

FIG. 2. First image showing gas entering the clay paste and time since initiation of injection (note varying times and textures in each test showing inconsistency of gas entry). (a) Test FVR_K01; ring highlights gas point of gas injection from beneath, (b) Test FVR_K02, (c) Test FVR_K03.

Figure 3 shows four images from Test FVR_K01, each being further along in the time of the test. A complex network of pathways develops from the point of initial entry, as shown in Fig. $2 a$. The width of pathways varies although the majority are narrow (on the millimetre scale of measurement). Pathways can be seen to split into branches, often at approximately $120^{\circ}$ to each other, as seen in Fig. 3b. A larger more significant pathway begins to develop and curve round in Fig. $3 c$. This becomes larger and the pathway widens in Fig. $3 d$. However, it stops propagating once it approaches another large pathway which has formed at the base of the images. The rate of the pathway network development seems to reduce after the initial establishment. The pathways are distributed over a large area of the paste; however there is not a clear pattern or predictable nature to the distribution.

Figure 4 shows four images from Test FVR_K02. Figure $4 a$ is the same image as Fig. $2 b$, where gas first enters the clay paste; from this point onwards the development of the pathway network in the clay paste can be seen. Figure $4 b$ shows the growth of pathways from this initial entry point. The pathways are narrow and angular, as seen in Test FVR_K01 (Fig. 3). Figure $4 c$ shows that after $399 \mathrm{~s}$ there is a more complex pattern to the pathways and they are well distributed, though still narrow and localized. The pattern of the pathways could be described as dendritic. As the test advances these pathways begin to develop at a much slower rate. The main change that can be seen at this time is a change in texture. Figure $4 e$ is a magnified portion of the same image as Fig. $4 d$. This shows a change in the texture of the clay paste within the pathway. The clay paste appears to be more wet and small bubbles can be seen within the texture of the clay. This is more clearly seen in the time-lapse video and can be interpreted as water being expelled from the clay paste as the gas pressure increased and the paste became compacted around the gas pathways.

Figure 5 shows the evolution of Test FVR_K03. Pathways can be seen to evolve from the initial gas entry point. The texture of the pathway developing above the original entry point appears to be wet and contain bubbles; this pathway propagates at a slower rate than the pathway which extends towards the bottom of the image. The pathways in this test develop at a faster rate than in Test FVR_K02 (Fig. 4), as shown by the time labels for each image. Compared to Tests FVR_K01 and FVR_K02 the pathways in this test are less distributed throughout the clay gouge. The pathway furthest to the left on Fig. $5 c$ propagates to the edge of the apparatus, though demonstration with only images is non-trivial. Once this pathway reaches the edge of the cell, gas breakthrough occurs, gas pressure reduces within the pathways and as a result some pathways appear to narrow and close. The most obvious pathway to fully close cuts down from the top of the image in Fig. $5 d$. It also appears that the pathways furthest from the breakthrough point may close first.

\section{Discussion}

The aim of this experimental programme was to visualize gas movement through clay-rich material. 
Initial gas entry and development of pathways

Localized pathway development

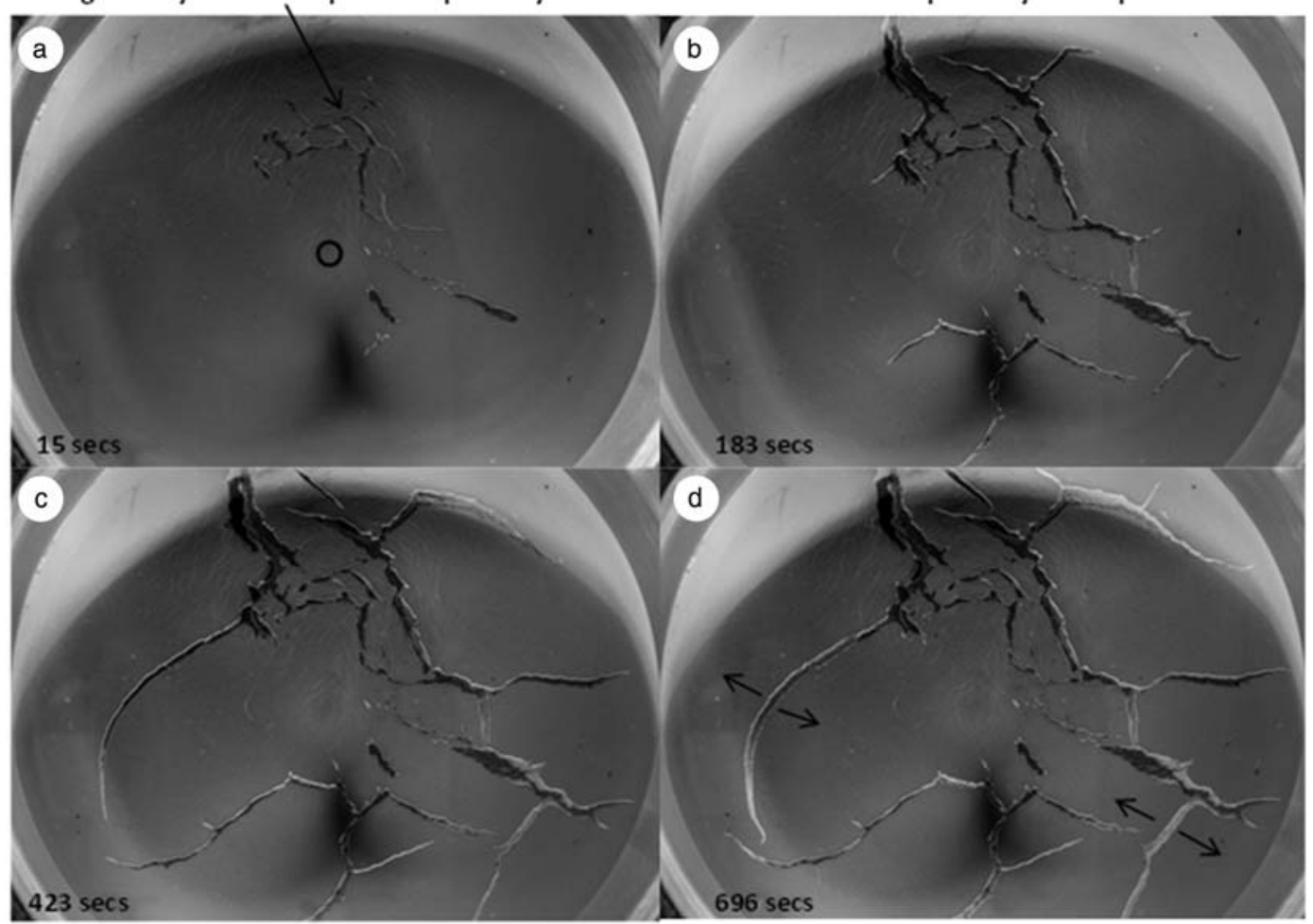

Complex network of narrow pathways

Dilation of pathways as they propagate, arrows show dilation direction.

FIG. 3. Evolution of Test FVR_K01 over time. (a) Initial gas entry, ring shows gas injection point, (b) Localized pathways begin to develop, (c) Development of network of pathways gives dendritic texture, $(d)$ Larger pathways can be seen to dilate as gas pressure increases, shown by arrows on image.

The experimental set-up has been shown to perform effectively and provides clear images of gas transport through clay whilst under stress. The results presented, and behaviour described, clearly demonstrate that the main transport mechanism for gas migration is via localized dilatant pathways.

Figure 2 shows the first image in which gas enters the clay paste in all three tests. The timings of these images can be considered to be the time at the point of gas entry into the system. The three images are dissimilar in appearance and gas pathway formation does not occur in the same place in any of the tests. This suggests that unexplained differences in localized clay properties dictate initial pathway formation. Once initial gas entry has occurred, pathway propagation exploits heterogeneities within the clay paste, which appear to be unavoidable as all tests are performed as identically as possible. The entry of gas into the clay paste occurs very suddenly, suggesting that gas entry occurs once the pressure overcomes the hydromechanical properties and stress state of the clay paste, as expected for clays (Graham et al., 2012). Behaviour such as this is similarly observed in laboratory experiments on other argillaceous materials (e.g. Cuss et al., 2014).

After the initial gas entry into the system, a complex network of pathways can be seen to develop in each test. The pathways are narrow and localized and can be seen to dilate as gas injection continues. The rate of propagation of these pathways occurs more rapidly towards the start and then appears to slow down. This is associated with a constant rate of gas injection. Therefore, as the pathways propagate there is a greater volume of space for the gas to fill. However, the injection rate remains constant, implying that gas pressure within 
Initial gas entry

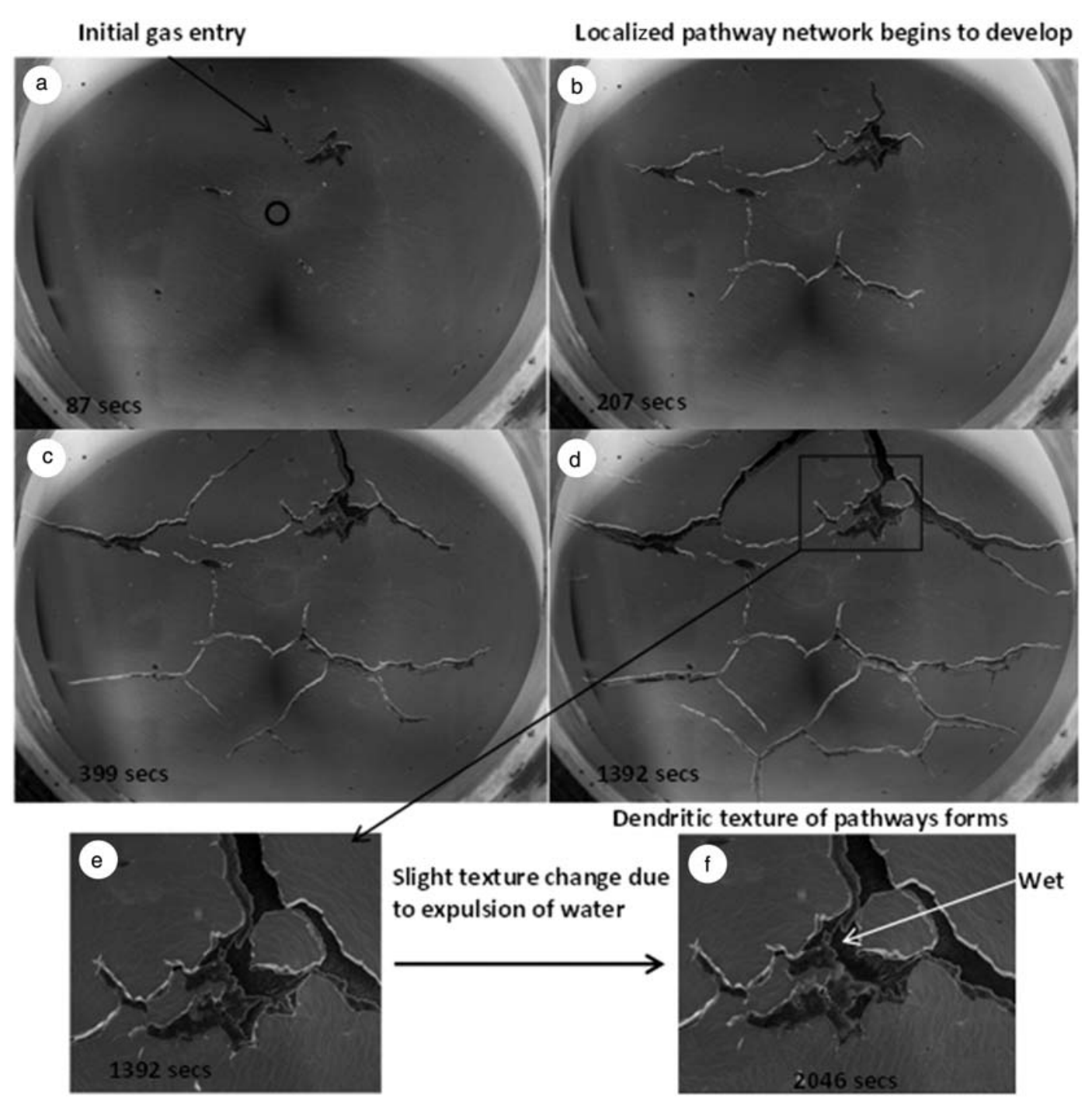

Localized pathway network begins to develop

\section{A. C. WISEALL ETAL.}

FIG. 4. Images showing evolution of pathways in Test 2. (a) Initial gas entry in isolated position, ring shows gas injection point; $(b, c, d)$ development of localized pathways in a well-distributed pattern; and $(e, f)$ zoomed-in images showing texture changes associated with water being expelled from the clay paste.

the pathways will not increase at the same rate whilst pathways propagate. It is also clear that multiple pathways develop simultaneously and that pathway formation is not dictated by a singular dominant pathway.

Figures $3 c$ and $d$ show a large curved pathway forming as the test proceeds. This stopped propagating as it approached another nearby pathway, indicating an interaction with the neighbouring pathways. Dilation causes compression of the surrounding clay paste; as a propagating pathway reaches this compressed zone of a nearby pathway, the propagating pathway becomes retarded and finds it more difficult to propagate.

Figures $4 d, e, f$ in Test FVR_K02 show a change in texture of the paste within a pathway. This is caused by de-watering of the clay paste; this is clearer in the time-lapse video of this test. The observed movement of water in the pathway is associated with the localized compression of the paste surrounding the pathways. This behaviour was postulated to occur by Cuss et al. (2014). 
Initial gas entry

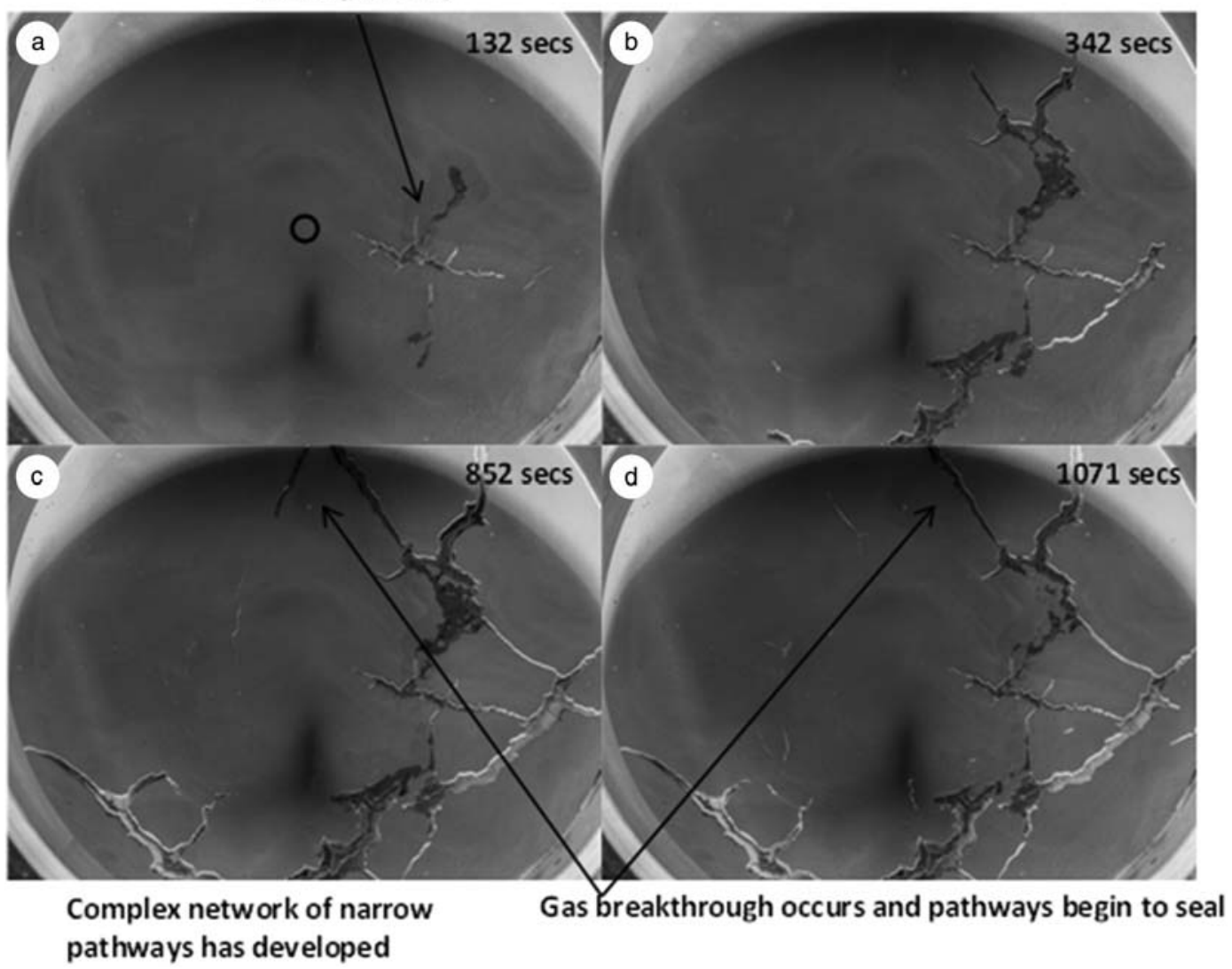

FIG. 5. Images showing evolution of Test FVR_K03 with respect to time. (a) Initial gas entry in isolated position, gas injection point shown by ring; $(b)$ development of dilatant pathways away from point of entry; $(c)$ large pathway to bottom left of image reaches edge of apparatus, resulting in gas breakthrough and decrease of gas pressure in pathways; and $(d)$ once gas breakthrough occurs, pathways away from point of breakthrough begin to close, as shown by label.

Figures $5 c$ and $d$ show the propagation of a pathway to the edge of the apparatus in Test FVR_K03. Once the pathway reaches the edge of the apparatus, this provides the gas with a preferential route for escape. This can be said to be the equivalent of gas breakthrough in more conventional laboratory experiments. Once a preferential pathway forms, the gas pressure within other pathways is relieved and closure occurs, as seen in Fig. 5. This behaviour has also been seen in other laboratory tests on clay-rich materials, such as the Boom Clay, and can be described as self-sealing behaviour (Harrington et al., 2012; Van Geet et al., 2008; Zhang et al., 2007). The pathways furthest from the point of gas breakthrough appear to close more completely and this may be due to a more rapid reduction in gas pressure.
The results presented here provide further evidence that the main transport mechanism for gas in clay-rich materials is via localized dilational pathways. This also further highlights the importance of hydro-mechanical coupling in the flow of gas in clay-rich materials. The understanding of gas transport through clay-rich materials is an important aspect of the safety case for the disposal of radioactive waste in a GDF.

\section{Conclusions and future work}

This study represents the initial results from tests carried out on a new apparatus developed at the BGS. It is clear that this apparatus has succeeded in its aim of visualizing the movement of gas through a 
clay-rich material. The results presented give further evidence to the theory that gas flow in clay occurs via localized dilatant pathways. This clearly demonstrates the propensity for high clay content to lead to the formation of localized dilatant pathways. These findings are consistent with reported observations of this mechanism in clay-rich rocks (Angeli et al., 2009; Autio et al., 2006; Cuss et al., 2014; Graham et al., 2012; Harrington et al., 2012). As the gas pressure increases and gas enters into the paste, a complex network of pathways can be seen to develop. As these pathways continue to develop and the gas pressure increases still further, the pathways can be seen to dilate. The surrounding paste is seen to compress as a result, forcing water from the saturated clay paste. Once gas breakthrough occurs, the gas pressure begins to decrease, resulting in the closure and self-sealing of pathways away from the point of breakthrough. Behaviour such as this has been detected experimentally in intact clay-rich material, but has not been directly observed in real time.

Understanding gas flow in clay-rich materials provides information to help guide repository design and predict future radionuclide migration. A significant uncertainty remains surrounding the processes governing gas flow in natural and engineered barrier systems; it is vital to understand these systems to an adequate level of detail to allow confidence in the assessment of site performance.

To further develop the results and interpretation presented in this paper, the experimental programme must be developed to incorporate more complex mineralogies and test histories. This will allow us to compare and contrast pathway development in different clay-rich materials. Furthermore, the apparatus may be developed to output more quantitative results (e.g. propagation rates). This novel testing methodology is providing new insight into the complex processes involved in advective gas migration within clay-rich materials.

\section{References}

Angeli, M., Soldal, M., Skurtveit, E. and Aker, E. (2009) Experimental percolation of supercritical $\mathrm{CO}_{2}$ through a caprock. Energy Procedia, 1, 3351-3358.

Autio, J., Gribi, P., Johnson, L. and Marschall, P. (2006) Effect of excavation damage zone on gas migration in a KBS-3H type repository at Olkiluotu. Physics and Chemistry of the Earth, 31, 649-653.

Besuelle, P., Viggiani, G., Desrues, J., Coll, C. and Charrier, P. (2013) A laboratory experimental study of thehydromechanical behaviour of Boom Clay. Rock Mechanics and Rock Engineering, 47, 143-155.
Chen, G.J., Maes, T., Vandervoort, F., Sillen, X., Van Marcke, P., Honty, M., Dierick, M. and Vanderniepen, P. (2014) Thermal impact on damaged Boom Clay and Opalinus Clay: Permeameter and isoastatic tests with $\mu \mathrm{CT}$ scanning. Rock Mechanics and Rock Engineering, 47, 87-99.

Cuss, R.J., Harrington, J., Giot, R. and Auvray, C. (2014) Experimental observations of mechanical dilation at the onset of gas flow in Callovo-Oxfordian claystone. Pp. 507-519 in: Clays in Natural and Engineered Barriers for Radioactive Waste Confinement, 400. Geological Society Special Publications, Geological Society of London, UK.

Graham, C.C., Harrington, J.F., Cuss, R.J. and Sellin, P., (2012) Gas migration experiments in bentonite: implications for numerical modelling. Mineralogical Magazine, 76, 3279-3292.

Harrington, J.F., Milodowski, A.E., Graham, C.C., Rushton, J.C. and Cuss, R.J. (2012) Evidence for gasinduced pathways in clay using a nano particle injection technique. Mineralogical Magazine, 76, 3327-3336.

Horseman, S.T., Harrington, J.F. and Sellin, P. (1999) Gas migration in clay barriers. Engineering Geology, 54, 139-149.

Marschall, P., Horseman, S. and Gimmi, T. (2005) Characterisation of gas transport properties of the Opalinus Clay; a potential host rock formation for radioactive waste disposal. Oil \& Gas Science and Technology - Review IFP, 60, 121-139.

Ortiz, L., Volckaert, G. and Mallants, D. (2002) Gas generation and migration in Boom Clay, a potential host rock formation for nuclear waste storage. Engineering Geology, 64, 287-296.

Sathar, S., Reeves, J.J., Cuss, R.J. and Harrington, J.F. (2012) The role of stress history on the flow of fluids through fractures. Mineralogical Magazine, 76, 3165-3177.

Van Geet, M., Bastiaens, W. and Ortiz, L. (2008) Selfsealing capacity of argillaceous rocks: review of laboratory results obtained from the SELFRAC project. Physics and Chemistry of the Earth, Parts A/ $B / C, 33, \mathrm{~S} 396-\mathrm{S} 406$.

Weetjens, E. and Sillen, X. (2006) Gas generation and migration in the near field of a supercontainerbaseddisposal system for vitrified high-level radioactive waste. Proceedings of the $11^{\text {th }}$ International High-Level Radioactive Waste Management Conference. IHLRWM, Las Vegas, Nevada, USA.

Wikramaratna, R.S., Goodfield, M., Rodwell, W.R, Nash, P.J. and Agg, P.J. (1993) A Preliminary Assessment of Gas Migration from the Copper/Steel Canister. SKB Technical report TR 93-31. Swedish Nuclear Fuel and Waste Management Company (SKB), Stockholm, Sweden.

Zhang, C-L, Rothfuchs, T., Su, K. and Hoteit, N. (2007) Experimental study of the thermo-hydro-mechanical behaviour of indurated clays. Physics and Chemistry of the Earth, 32, 957-965. 